medRxiv preprint doi: https://doi.org/10.1101/2021.01.29.21250407; this version posted February 1, 2021. The copyright holder for this preprint (which was not certified by peer review) is the author/funder, who has granted medRxiv a license to display the preprint in perpetuity.

It is made available under a CC-BY-ND 4.0 International license .

\title{
12-lead Electrocardiogram in Hospitalized COVID 19 Patients
}

Mohamed Shokr ${ }^{1}$, Omar Chehab ${ }^{2}$, Mustafa Ajam ${ }^{3}$, Manmohan Singh $^{1}$, Said Ashraf ${ }^{1}$, John Dawdy ${ }^{1}$, Mohit Pahuja $^{1}$, Vivek Reddy ${ }^{1}$, Ahmed Subahi ${ }^{1}$, M. Chadi Alraies ${ }^{1}$, Luis Afonso ${ }^{1}$, Randy Lieberman ${ }^{1}$

1: Department of Cardiology, Wayne State University, Detroit, MI, USA

2: Department of Internal Medicine, Wayne State University, Detroit, MI, USA

3: Department of Pulmonary and Critical Care Medicine, Oregon Health and Science University, Portland, OR, USA

Corresponding Author Information:

Mohamed Shokr, MD

Phone: (313) 244-7083

Email: Mohamed.Shokr@nyulangone.org

\begin{abstract}
COVID-19 pandemic resulted in considerable morbidity and mortality. We analyzed 345 Electrocardiograms of 100 COVID-19 patients admitted to our tertiary care center in Detroit, during the initial month of Covid-19. Findings were correlated with mortality, cardiac injury and inflammatory markers. Our cohort included 61\% males and 77\% African Americans. The median age and BMI were 66 years (57-74) and $31 \mathrm{~kg} / \mathrm{m}^{2}$ (26.1-39), respectively. We observed atrial arrhythmias in $29 \%$ of the patients (17\% new onset), First degree heart block in 12\%, ST-T segment changes in 17\%, S1Q3T3 pattern in $19 \%$, premature ventricular complexes in $23 \%$, premature atrial complexes in $13 \%$, Q waves in $27 \%, T$ wave inversion in $42 \%$ of the cases. While presence of premature atrial complexes or left atrial abnormality correlated with mortality $(P=0.02 \& 0.03$, respectively), other findings did not show significant correlation in this small cohort of patients.
\end{abstract}


medRxiv preprint doi: https://doi.org/10.1101/2021.01.29.21250407; this version posted February 1, 2021. The copyright holder for this preprint (which was not certified by peer review) is the author/funder, who has granted medRxiv a license to display the preprint in perpetuity.

It is made available under a CC-BY-ND 4.0 International license .

\section{Manuscript}

COVID-19 pandemic resulted in considerable morbidity and mortality (1). We analyzed 345 Electrocardiograms of 100 COVID-19 patients admitted to our tertiary care center in Detroit, during the initial month of Covid-19. Findings were correlated with mortality, cardiac injury and inflammatory markers (Tables 1, 2S \&3S supplementary index).

For our analysis, categorical and continuous variables were reported as frequency or percentage and mean \pm standard error (SE) or median \pm interquartile range, based on the normality of the data. Baseline demographics and comorbidities between groups were compared using the Pearson $\chi^{2}$ test for categorical variables and one-way linear regression for continuous variables (Table 4S supplementary index)

Our cohort included $61 \%$ males and 77\% African Americans. The median age and BMI were 66 years (57$74)$ and $31 \mathrm{~kg} / \mathrm{m}^{2}$ (26.1-39), respectively. History of Hypertension, Diabetes Mellitus, Cancer and lung disease was present in $71 \%, 40 \%, 15 \%$ and $26 \%$ of the patients, respectively. $12 \%$ had coronary artery disease, $10 \%$ had Systolic heart failure and $12 \%$ had history of atrial arrhythmias at baseline. The median hospital stay was 10.4 days (6.7-16) with $48 \%$ mortality.

We observed atrial arrhythmias in $29 \%$ of the patients ( $17 \%$ new onset ), First degree heart block in $12 \%$ (6\% were transient), ST-T segment changes in 17\%, S1Q3T3 pattern in 19\%, Premature ventricular complexes (PVCs) in 23\%, premature atrial complexes( PACs) in 13\%, Q waves in 27\%, T wave inversion in $42 \%$ of the cases. Long QTc ( $>470$ in males, $>480$ in females) was noted in 37 cases, of whom 20 patients were on Hydroxychloroquine and Azithromycin. There was no correlation between long QTC and mortality $(\mathrm{P}=0.5)$.

Myocardial injury due to hypoxia and direct viral tissue invasion, sympathetic hyperactivity secondary to the cytokine storm and drug induced QTc prolongation are postulated mechanism of arrhythmias in COVID-19 cases (2)

Transient complete heart block was previously reported $(3,4)$.In our cohort, only first degree heart block was present without higher grade block.

S1Q3T3 pattern, possibly due to cor pulmonale, was reported in 2 Covid-19 cases (4). It was noted in $19 \%$ of patients in our cohort and did not correlate with mortality $(P=0.4)$

ST elevation without obstructive coronary artery disease was reported in COVID-19 patients (5). We noted 8 cases of ST elevation, of which 5 were transient and 8 cases of ST depression. None of these patients underwent coronary angiogram during hospitalization. ST segment changes did not correlate with mortality $(P=0.5)$

Premature complexes are possibly explained by the higher sympathetic tone, vasopressors use, electrolyte abnormalities and myocarditis. Right ventricular outflow tract was the most common site of origin (47\% of cases). While PACs correlated with mortality ( $\mathrm{P}=0.02)$, PVCs correlation didn't reach 
medRxiv preprint doi: https://doi.org/10.1101/2021.01.29.21250407; this version posted February 1, 2021. The copyright holder for this preprint (which was not certified by peer review) is the author/funder, who has granted medRxiv a license to display the preprint in perpetuity.

It is made available under a CC-BY-ND 4.0 International license .

statistical significance $(P=0.05)$. EKG abnormalities are prevalent in this cohort of COVID-19 patients and can be a helpful diagnostic tool for underlying cardiac pathologies.

\section{Acknowledgements:}

None.

\section{Financial Disclosures:}

Authors have no financial disclosures to report.

\section{Conflict of Interest:}

The authors declare that they have no conflict of interest concerning this article.

\section{Informed Consent:}

Not applicable.

\section{Authors Contributions:}

Shokr M designed the research, collected the data and wrote the manuscript. Chehab $\mathrm{O}$ analyzed the data. All authors contributed to data collection. Shokr M and Lieberman R approved the final version of the manuscript.

\section{References:}

1- Shi S, Qin M, Shen B, Cai Y, Liu T, Yang F, Gong W, Liu X, Liang J, Zhao Q, Huang H. Association of cardiac injury with mortality in hospitalized patients with COVID-19 in Wuhan, China. JAMA cardiology. 2020 Mar 25.

2- Lazzerini PE, Boutjdir M, Capecchi PL. COVID-19, Arrhythmic Risk and Inflammation: Mind the Gap!. Circulation. 2020 Apr 14. 
medRxiv preprint doi: https://doi.org/10.1101/2021.01.29.21250407; this version posted February 1, 2021. The copyright holder for this preprint (which was not certified by peer review) is the author/funder, who has granted medRxiv a license to display the preprint in perpetuity.

It is made available under a CC-BY-ND 4.0 International license.

3- Azarkish M, Eslami M, Mollazadeh R. Transient complete heart block in a patient with critical COVID-19. European Heart Journal. 2020 Apr 14.

4- He J, Wu B, Chen Y, Tang J, Liu Q, Zhou S, Chen C, Qin Q, Huang K, Lv J,Chen Y, Peng D, Characteristic ECG Manifestations in Patients with COVID-19, Canadian Journal of Cardiology (2020), doi: https://doi.org/10.1016/j.cjca.2020.03.028.

5- Bangalore S, Sharma A, Slotwiner A, Yatskar L, Harari R, Shah B, Ibrahim H, Friedman GH, Thompson C, Alviar CL, Chadow HL. ST-segment elevation in patients with Covid-19-A case series. New England Journal of Medicine. 2020 Apr 17.

Table 1: Correlation of Electrocardiogram (EKG) findings with mortality, intensive care unit (ICU) admission, Cardiac injury ( High sensitivity(Hs) Troponin $>17 \mathrm{ng} / \mathrm{L}$ ) and Median C-reactive protein (175 $\mathrm{mg} / \mathrm{L}$ )

\begin{tabular}{|c|c|c|c|c|c|c|c|c|c|c|c|c|c|}
\hline \multirow[t]{2}{*}{ EKG finding } & \multirow[t]{2}{*}{ Prevalence } & \multicolumn{2}{|c|}{ Survival } & \multirow[t]{2}{*}{ P-value } & \multicolumn{2}{|c|}{ ICU admission } & \multirow[t]{2}{*}{ P-value } & \multicolumn{2}{|c|}{ Hs Troponin } & \multirow[t]{2}{*}{ P-value } & \multicolumn{2}{|c|}{ Median CRP } & \multirow[t]{2}{*}{ P-value } \\
\hline & & yes & No & & No & Yes & & $\leq 17$ & $>17$ & & $<175$ & $\geq 175$ & \\
\hline Transient $1^{\text {st }}{ }^{\circ} \mathrm{HB}$ & $6 \%$ & $1.9 \%$ & $10.4 \%$ & \multirow[t]{2}{*}{0.2} & $10.8 \%$ & $3.2 \%$ & \multirow[t]{2}{*}{0.055} & $9.5 \%$ & $6.4 \%$ & \multirow[t]{2}{*}{0.7} & $9.1 \%$ & $2.3 \%$ & \multirow[t]{2}{*}{0.2} \\
\hline Persistent $1{ }^{\text {st }}{ }^{\circ} \mathrm{HB}$ & $6 \%$ & $5.8 \%$ & $6.3 \%$ & & $0.0 \%$ & $9.5 \%$ & & $4.8 \%$ & $2.1 \%$ & & $4.5 \%$ & $9.1 \%$ & \\
\hline$T$ wave inversion & $42 \%$ & $46.2 \%$ & $37.5 \%$ & 0.2 & $43.2 \%$ & $41.3 \%$ & 0.5 & $61.9 \%$ & $36.2 \%$ & 0.04 & $36.4 \%$ & $52.3 \%$ & 0.09 \\
\hline $\begin{array}{l}\text { Premature ventricular } \\
\text { complexes }\end{array}$ & $23 \%$ & $15.4 \%$ & $31.3 \%$ & 0.050 & $21.6 \%$ & $23.8 \%$ & 0.5 & $14.3 \%$ & $23.4 \%$ & 0.3 & $25.0 \%$ & $18.2 \%$ & 0.3 \\
\hline Premature atrial & $13 \%$ & $5.8 \%$ & $20.8 \%$ & 0.02 & $8.1 \%$ & $15.9 \%$ & 0.2 & $4.8 \%$ & $12.8 \%$ & 0.3 & $15.9 \%$ & $9.1 \%$ & 0.3 \\
\hline
\end{tabular}


medRxiv preprint doi: https://doi.org/10.1101/2021.01.29.21250407; this version posted February 1, 2021. The copyright holder for this preprint (which was not certified by peer review) is the author/funder, who has granted medRxiv a license to display the preprint in perpetuity.

It is made available under a CC-BY-ND 4.0 International license .

\begin{tabular}{|c|c|c|c|c|c|c|c|c|c|c|c|c|c|}
\hline complexes & & & & & & & & & & & & & \\
\hline \multicolumn{4}{|l|}{ Rhythm on admission } & \multirow[t]{4}{*}{0.9} & & & \multirow[t]{4}{*}{0.059} & & & \multirow[t]{4}{*}{0.3} & & & \multirow[t]{4}{*}{0.2} \\
\hline Sinus rhythm & $56 \%$ & $57.7 \%$ & $54.2 \%$ & & $70.3 \%$ & $47.6 \%$ & & $66.7 \%$ & $46.8 \%$ & & $61.4 \%$ & $45.5 \%$ & \\
\hline Sinus Tachycardia & $33 \%$ & $30.8 \%$ & $35.4 \%$ & & $18.9 \%$ & $41.3 \%$ & & $28.6 \%$ & $42.6 \%$ & & $27.3 \%$ & $43.2 \%$ & \\
\hline Atrial fibrillation & $11 \%$ & $11.5 \%$ & $10.4 \%$ & & $10.8 \%$ & $11.1 \%$ & & $4.8 \%$ & $10.6 \%$ & & $11.4 \%$ & $11.4 \%$ & \\
\hline \multicolumn{14}{|l|}{ Rhythm during admission } \\
\hline Sinus bradycardia & $13 \%$ & $17.3 \%$ & $8.3 \%$ & 0.1 & $24.3 \%$ & $6.3 \%$ & 0.01 & $9.5 \%$ & $10.6 \%$ & 0.6 & $18.2 \%$ & $9.1 \%$ & 0.1 \\
\hline Sinus tachycardia & $81 \%$ & $78.8 \%$ & $83.3 \%$ & 0.4 & $67.6 \%$ & $88.9 \%$ & 0.01 & $90.5 \%$ & $80.9 \%$ & 0.3 & $72.7 \%$ & $90.9 \%$ & 0.02 \\
\hline Left atrial abnormality & $5.1 \%$ & $9.8 \%$ & $0 \%$ & 0.03 & $5.6 \%$ & $4.8 \%$ & 0.6 & $0.0 \%$ & $4.3 \%$ & 0.5 & $2.4 \%$ & $9.1 \%$ & 0.1 \\
\hline Q waves & & & & \multirow[t]{4}{*}{0.5} & & & \multirow[t]{4}{*}{0.02} & & & \multirow[t]{4}{*}{0.6} & & & \multirow[t]{4}{*}{0.1} \\
\hline Q waves (Inferior) & $22 \%$ & $21.2 \%$ & $22.9 \%$ & & $21.6 \%$ & $22.2 \%$ & & $23.8 \%$ & $25.5 \%$ & & $18.2 \%$ & $29.5 \%$ & \\
\hline (Anterior) & $2 \%$ & $3.8 \%$ & $0 \%$ & & $5.4 \%$ & $0.0 \%$ & & $0.0 \%$ & $2.1 \%$ & & $2.3 \%$ & $0.0 \%$ & \\
\hline$\geq 2$ territories & $3 \%$ & $3.8 \%$ & $2.1 \%$ & & $8.1 \%$ & $0.0 \%$ & & $0.0 \%$ & $6.4 \%$ & & $6.8 \%$ & $0.0 \%$ & \\
\hline Low voltage & $16 \%$ & $9.6 \%$ & $22.9 \%$ & 0.06 & $13.5 \%$ & $17.5 \%$ & 0.4 & $14.3 \%$ & $14.9 \%$ & 0.6 & $18.2 \%$ & $11.4 \%$ & 0.27 \\
\hline LVH & $15 \%$ & $17.3 \%$ & $12.5 \%$ & 0.3 & $16.2 \%$ & $14.3 \%$ & 0.5 & $9.5 \%$ & $19.1 \%$ & 0.3 & $6.8 \%$ & $25.0 \%$ & 0.01 \\
\hline Prolonged QTC & $37 \%$ & $36.5 \%$ & $37.5 \%$ & 0.5 & $29.7 \%$ & $41.3 \%$ & 0.2 & $42.9 \%$ & $34.0 \%$ & 0.3 & $31.8 \%$ & $45.5 \%$ & 0.1 \\
\hline S1Q3T3 & $19 \%$ & $17.3 \%$ & $20.8 \%$ & 0.4 & $24.3 \%$ & $15.9 \%$ & 0.2 & $23.8 \%$ & $25.5 \%$ & 0.6 & $18.2 \%$ & $22.7 \%$ & 0.3 \\
\hline
\end{tabular}

Table 2S: Correlation of EKG findings with Mortality, ICU admission, Cardiac injury and elevated Brain Natriuretic peptide (BNP) level ( $>100 \mathrm{pg} / \mathrm{ml}$ )

\begin{tabular}{|c|c|c|c|c|c|c|c|c|c|c|c|c|c|}
\hline \multirow[t]{2}{*}{ EKG finding } & \multirow[t]{2}{*}{$\mathrm{N}=100$} & \multicolumn{2}{|c|}{ Survivors } & \multirow[t]{2}{*}{ P-value } & \multicolumn{2}{|c|}{ ICU admission } & \multirow[t]{2}{*}{ P-value } & \multicolumn{2}{|c|}{ Cardiac injury } & \multirow[t]{2}{*}{ P-value } & \multicolumn{2}{|c|}{ BNP } & \multirow[t]{2}{*}{ P-value } \\
\hline & & yes & No & & No & Yes & & No & Yes & & $\leq 100$ & $>100$ & \\
\hline $1^{\text {st }}$ degree heart block (HB) & $12 \%$ & $7.7 \%$ & $16.7 \%$ & 0.1 & $10.8 \%$ & $12.7 \%$ & 0.5 & $14.3 \%$ & $8.5 \%$ & 0.37 & $11.8 \%$ & $12.5 \%$ & 0.7 \\
\hline Transient $1^{\mathrm{ST}}$ degree HB & $6 \%$ & $1.9 \%$ & $10.4 \%$ & 0.2 & $10.8 \%$ & $3.2 \%$ & \multirow[t]{2}{*}{0.055} & $9.5 \%$ & $6.4 \%$ & \multirow[t]{2}{*}{0.7} & $5.9 \%$ & $4.2 \%$ & \multirow[t]{2}{*}{0.9} \\
\hline Persistent $1^{\text {st }}$ degree HB & $6 \%$ & $5.8 \%$ & $6.3 \%$ & & $0.0 \%$ & $9.5 \%$ & & $4.8 \%$ & $2.1 \%$ & & $5.9 \%$ & $8.3 \%$ & \\
\hline Transient ST elevation & $5 \%$ & $3.8 \%$ & $6.3 \%$ & \multirow[t]{3}{*}{0.4} & $2.7 \%$ & $6.3 \%$ & \multirow[t]{3}{*}{0.5} & $4.8 \%$ & $6.4 \%$ & \multirow[t]{3}{*}{0.4} & $5.9 \%$ & 4.25 & \multirow[t]{3}{*}{0.7} \\
\hline Persistent ST elevation & $3 \%$ & $1.9 \%$ & $4.2 \%$ & & $0.0 \%$ & $4.8 \%$ & & $4.8 \%$ & $2.1 \%$ & & $5.9 \%$ & $0.0 \%$ & \\
\hline ST depression & $8 \%$ & $11.5 \%$ & $4.2 \%$ & & $8.1 \%$ & $7.9 \%$ & & $14.3 \%$ & $6.4 \%$ & & $11.8 \%$ & $8.3 \%$ & \\
\hline
\end{tabular}


medRxiv preprint doi: https://doi.org/10.1101/2021.01.29.21250407; this version posted February 1, 2021. The copyright holder for this preprint (which was not certified by peer review) is the author/funder, who has granted medRxiv a license to display the preprint in perpetuity.

It is made available under a CC-BY-ND 4.0 International license.

\begin{tabular}{|c|c|c|c|c|c|c|c|c|c|c|c|c|c|}
\hline Early repolarization & $1 \%$ & $1.9 \%$ & $0 \%$ & & $2.7 \%$ & $0.0 \%$ & & $4.8 \%$ & $0.0 \%$ & & $0.0 \%$ & $4.2 \%$ & \\
\hline T wave inversion & $42 \%$ & $46.2 \%$ & $37.5 \%$ & 0.2 & $43.2 \%$ & $41.3 \%$ & 0.5 & $61.9 \%$ & $36.2 \%$ & 0.04 & $41.2 \%$ & $50.0 \%$ & 0.4 \\
\hline T wave inversion (inferior) & $5 \%$ & $3.8 \%$ & $6.3 \%$ & \multirow[t]{4}{*}{0.5} & $2.7 \%$ & $6.3 \%$ & \multirow[t]{4}{*}{0.4} & $4.8 \%$ & $6.4 \%$ & \multirow[t]{4}{*}{0.04} & $0.0 \%$ & $12.5 \%$ & \multirow[t]{4}{*}{0.4} \\
\hline (anterior) & $18 \%$ & $21.2 \%$ & $14.6 \%$ & & $16.2 \%$ & $19.0 \%$ & & $14.3 \%$ & $19.1 \%$ & & $23.5 \%$ & $25.0 \%$ & \\
\hline (Lateral) & $2 \%$ & $3.8 \%$ & $0 \%$ & & $5.4 \%$ & $0.0 \%$ & & $4.8 \%$ & $2.1 \%$ & & $5.9 \%$ & $0.0 \%$ & \\
\hline ( $\geq 2$ territories) & $17 \%$ & $17.3 \%$ & $16.7 \%$ & & $18.9 \%$ & $15.9 \%$ & & $38.1 \%$ & $8.5 \%$ & & $11.8 \%$ & $12.5 \%$ & \\
\hline S1Q3T3 & $19 \%$ & $17.3 \%$ & $20.8 \%$ & 0.4 & $24.3 \%$ & $15.9 \%$ & 0.2 & $23.8 \%$ & $25.5 \%$ & 0.6 & $29.4 \%$ & $25.0 \%$ & 0.5 \\
\hline Premature ventricular complexes & $23 \%$ & $15.4 \%$ & $31.3 \%$ & 0.050 & $21.6 \%$ & $23.8 \%$ & 0.5 & $14.3 \%$ & $23.4 \%$ & 0.3 & $29.4 \%$ & $8.3 \%$ & 0.1 \\
\hline Premature atrial complexes & $13 \%$ & $5.8 \%$ & $20.8 \%$ & 0.02 & $8.1 \%$ & $15.9 \%$ & 0.2 & $4.8 \%$ & $12.8 \%$ & 0.3 & $23.5 \%$ & $4.2 \%$ & 0.1 \\
\hline Poor $\mathrm{R}$ wave progression & $14 \%$ & $17.3 \%$ & $10.4 \%$ & 0.2 & $18.9 \%$ & $11.1 \%$ & 0.2 & $14.3 \%$ & $14.9 \%$ & 0.6 & $5.9 \%$ & $16.7 \%$ & 0.3 \\
\hline \multicolumn{14}{|l|}{ Rhythm on admission } \\
\hline Sinus rhythm & $56 \%$ & $57.7 \%$ & $54.2 \%$ & \multirow[t]{3}{*}{0.9} & $70.3 \%$ & $47.6 \%$ & \multirow[t]{3}{*}{0.059} & $66.7 \%$ & $46.8 \%$ & \multirow[t]{3}{*}{0.3} & $52.9 \%$ & $58.3 \%$ & \multirow[t]{3}{*}{0.9} \\
\hline Sinus tachycardia & $33 \%$ & $30.8 \%$ & $35.4 \&$ & & $18.9 \%$ & $41.3 \%$ & & $28.6 \%$ & $42.6 \%$ & & $35.3 \%$ & $29.2 \%$ & \\
\hline Atrial fibrillation & $11 \%$ & $11.5 \%$ & $10.4 \%$ & & $10.8 \%$ & $11.1 \%$ & & $4.8 \%$ & $10.6 \%$ & & $11.8 \%$ & $12.5 \%$ & \\
\hline \multicolumn{14}{|l|}{ Rhythm during admission } \\
\hline Atrial arrhythmias & $29 \%$ & $17.3 \%$ & $27.1 \%$ & 0.2 & $16.2 \%$ & $25.4 \%$ & 0.2 & $19.0 \%$ & $25.5 \%$ & 0.4 & $23.5 \%$ & $25.0 \%$ & 0.6 \\
\hline Atrial fibrillation & $18 \%$ & $15.4 \%$ & $20.8 \%$ & 0.3 & $13.5 \%$ & $20.6 \%$ & 0.3 & $14.3 \%$ & $23.4 \%$ & 0.3 & $23.5 \%$ & $20.8 \%$ & 0.6 \\
\hline Atrial flutter & $6 \%$ & $5.8 \%$ & $6.3 \%$ & 0.6 & $5.4 \%$ & $6.3 \%$ & 0.6 & $4.8 \%$ & $2.1 \%$ & 0.5 & $5.9 \%$ & $8.3 \%$ & 0.6 \\
\hline Atrial tachycardia & $5 \%$ & $1.9 \%$ & $8.3 \%$ & 0.5 & $2.7 \%$ & $3.2 \%$ & 0.7 & $4.8 \%$ & $4.3 \%$ & 0.7 & $0.0 \%$ & $4.2 \%$ & 0.6 \\
\hline Sinus bradycardia & $13 \%$ & $17.3 \%$ & $8.3 \%$ & 0.1 & $24.3 \%$ & $6.3 \%$ & 0.01 & $9.5 \%$ & $10.6 \%$ & 0.6 & $5.9 \%$ & $16.7 \%$ & 0.3 \\
\hline Sinus tachycardia & $81 \%$ & $78.8 \%$ & $83.3 \%$ & 0.4 & $67.6 \%$ & $88.9 \%$ & 0.01 & $90.5 \%$ & $80.9 \%$ & 0.3 & $82.4 \%$ & $75.0 \%$ & 0.4 \\
\hline Left atrial abnormality & $5.1 \%$ & $9.8 \%$ & $0 \%$ & 0.03 & $5.6 \%$ & $4.8 \%$ & 0.6 & $0.0 \%$ & $4.3 \%$ & 0.5 & $12.5 \%$ & $4.2 \%$ & 0.3 \\
\hline Right atrial abnormality & $7.1 \%$ & $5.9 \%$ & $8.5 \%$ & 0.4 & $5.6 \%$ & $8.1 \%$ & 0.5 & $5.0 \%$ & $8.7 \%$ & 0.5 & $6.3 \%$ & $12.5 \%$ & 0.5 \\
\hline Left axis deviation & $24.2 \%$ & $21.6 \%$ & $27.1 \%$ & \multirow[t]{2}{*}{0.8} & $27.0 \%$ & $22.6 \%$ & \multirow[t]{2}{*}{0.8} & $19.0 \%$ & $27.7 \%$ & \multirow[t]{2}{*}{0.4} & $17.6 \%$ & $33.3 \%$ & \multirow[t]{2}{*}{0.2} \\
\hline Right axis deviation & $2.1 \%$ & $2 \%$ & $2.1 \%$ & & $2.7 \%$ & $1.6 \%$ & & $0.0 \%$ & $4.3 \%$ & & $0.0 \%$ & $0.0 \%$ & \\
\hline Right bundle branch block (RBBB) & $4 \%$ & $3.8 \%$ & $4.2 \%$ & 0.7 & $5.4 \%$ & $3.2 \%$ & 0.5 & $4.8 \%$ & $4.3 \%$ & 0.7 & $5.9 \%$ & $0.0 \%$ & 0.4 \\
\hline Incomplete RBBB & $3 \%$ & $3.8 \%$ & $2.1 \%$ & 0.5 & $0.0 \%$ & $4.8 \%$ & 0.2 & $0.0 \%$ & $2.1 \%$ & 0.7 & $0.0 \%$ & $4.2 \%$ & 0.6 \\
\hline Qwaves & $27 \%$ & $28.8 \%$ & $25 \%$ & 0.4 & $35.1 \%$ & $22.2 \%$ & 0.1 & $23.8 \%$ & $34.0 \%$ & 0.3 & $35.3 \%$ & $37.5 \%$ & 0.6 \\
\hline Inferior Q waves & $22 \%$ & $21.2 \%$ & $22.9 \%$ & \multirow[t]{3}{*}{0.5} & $21.6 \%$ & $22.2 \%$ & \multirow[t]{3}{*}{0.02} & $23.8 \%$ & $25.5 \%$ & \multirow[t]{3}{*}{0.6} & $29.4 \%$ & $29.2 \%$ & \multirow[t]{3}{*}{0.8} \\
\hline Anterior Q waves & $2 \%$ & $3.8 \%$ & $0 \%$ & & $5.4 \%$ & $0.0 \%$ & & $0.0 \%$ & $2.1 \%$ & & $5.9 \%$ & $4.2 \%$ & \\
\hline Q waves in $\geq 2$ territories & $3 \%$ & $3.8 \%$ & $2.1 \%$ & & $8.1 \%$ & $0.0 \%$ & & $0.0 \%$ & $6.4 \%$ & & $0.0 \%$ & $4.2 \%$ & \\
\hline Low voltage & $16 \%$ & $9.6 \%$ & $22.9 \%$ & 0.06 & $13.5 \%$ & $17.5 \%$ & 0.4 & $14.3 \%$ & $14.9 \%$ & 0.6 & $5.9 \%$ & $29.2 \%$ & 0.06 \\
\hline Left ventricular hypertrophy & $15 \%$ & $17.3 \%$ & $12.5 \%$ & 0.3 & $16.2 \%$ & $14.3 \%$ & 0.5 & $9.5 \%$ & $19.1 \%$ & 0.3 & $17.6 \%$ & $12.5 \%$ & 0.5 \\
\hline Left anterior fascicular block & $12 \%$ & $13.5 \%$ & $10.4 \%$ & 0.4 & $18.9 \%$ & $7.9 \%$ & 0.09 & $9.5 \%$ & $17.0 \%$ & 0.3 & $5.9 \%$ & $20.8 \%$ & 0.2 \\
\hline Bi-fascicular block & $4 \%$ & $3.8 \%$ & $4.2 \%$ & 0.7 & $5.4 \%$ & $3.2 \%$ & 0.5 & $4.8 \%$ & $4.3 \%$ & 0.7 & $5.9 \%$ & $0.0 \%$ & 0.4 \\
\hline Normal QRS width & $95 \%$ & $94.2 \%$ & $95.8 \%$ & 0.5 & $94.6 \%$ & $95.2 \%$ & 0.6 & $95.2 \%$ & $95.7 \%$ & 0.67 & $94.1 \%$ & $100 \%$ & 0.4 \\
\hline
\end{tabular}

Table 3S: Correlation of EKG findings with median D-dimer of $1 \mathrm{mg} / \mathrm{L}$, median C-reactive protein (CRP) of $175 \mathrm{mg} / \mathrm{l}$, median Ferritin level of $900 \mathrm{ng} / \mathrm{ml}$ and median Length of stay (LOS) of 10 days

\begin{tabular}{|c|c|c|c|c|c|c|c|c|c|c|c|c|}
\hline \multirow[t]{2}{*}{ EKG finding } & \multicolumn{2}{|c|}{ D-Dimer } & \multirow[t]{2}{*}{ P-value } & \multicolumn{2}{|c|}{ CRP } & \multirow[t]{2}{*}{ P-value } & \multicolumn{2}{|c|}{ Ferritin } & \multirow[t]{2}{*}{ P-value } & \multicolumn{2}{|c|}{ LOS } & \multirow[t]{2}{*}{ P-value } \\
\hline & $<1$ & $\geq 1$ & & $<175$ & $\geq 175$ & & $<900$ & $\geq 900$ & & $<10$ & $\geq 10$ & \\
\hline $1^{\text {st }}$ degree heart block $(\mathrm{HB})$ & $6.3 \%$ & $11.5 \%$ & 0.47 & $13.6 \%$ & $11.4 \%$ & 0.5 & $13.6 \%$ & $12.2 \%$ & 0.5 & $10.6 \%$ & $13.2 \%$ & 0.46 \\
\hline Transient $1^{\text {ST }}$ degree HB & $0.0 \%$ & $7.7 \%$ & \multirow[t]{2}{*}{0.4} & $9.1 \%$ & $2.3 \%$ & \multirow[t]{2}{*}{0.2} & $9.1 \%$ & $4.1 \%$ & \multirow[t]{2}{*}{0.5} & $6.4 \%$ & $5.7 \%$ & \multirow[t]{2}{*}{0.7} \\
\hline Persistent $1^{\text {st }}$ degree HB & $6.3 \%$ & $3.8 \%$ & & $4.5 \%$ & $9.1 \%$ & & $4.5 \%$ & $8.2 \%$ & & $4.3 \%$ & $7.5 \%$ & \\
\hline Transient ST elevation & $0.0 \%$ & $7.7 \%$ & \multirow[t]{4}{*}{0.7} & $2.3 \%$ & $6.8 \%$ & \multirow[t]{4}{*}{0.7} & $2.3 \%$ & $8.2 \%$ & \multirow[t]{4}{*}{0.5} & $4.3 \%$ & $5.7 \%$ & \multirow[t]{4}{*}{0.7} \\
\hline Persistent ST elevation & $6.3 \%$ & $3.8 \%$ & & $2.3 \%$ & $2.3 \%$ & & $2.3 \%$ & $4.1 \%$ & & $2.1 \%$ & $3.8 \%$ & \\
\hline ST depression & $6.3 \%$ & $9.6 \%$ & & $9.1 \%$ & $9.1 \%$ & & $9.1 \%$ & $8.2 \%$ & & $6.4 \%$ & $9.4 \%$ & \\
\hline Early repolarization & $0.0 \%$ & $1.9 \%$ & & $2.3 \%$ & $0.0 \%$ & & $2.3 \%$ & $0.0 \%$ & & $2.1 \%$ & $0.0 \%$ & \\
\hline T wave inversion & $37.5 \%$ & $48.1 \%$ & 0.3 & $36.4 \%$ & $52.3 \%$ & 0.09 & $36.4 \%$ & $51.0 \%$ & 0.1 & $46.8 \%$ & $37.7 \%$ & 0.2 \\
\hline T wave inversion (Inferior) & $0.0 \%$ & $5.8 \%$ & 0.7 & $2.3 \%$ & $9.1 \%$ & 0.2 & $2.3 \%$ & $8.2 \%$ & 0.3 & $4.3 \%$ & $5.7 \%$ & 0.1 \\
\hline
\end{tabular}


medRxiv preprint doi: https://doi.org/10.1101/2021.01.29.21250407; this version posted February 1, 2021. The copyright holder for this preprint (which was not certified by peer review) is the author/funder, who has granted medRxiv a license to display the preprint in perpetuity.

It is made available under a CC-BY-ND 4.0 International license.

\begin{tabular}{|c|c|c|c|c|c|c|c|c|c|c|c|c|}
\hline (Anterior) & $18.8 \%$ & $19.2 \%$ & & $15.9 \%$ & $20.5 \%$ & & $15.9 \%$ & $20.4 \%$ & & $12.8 \%$ & $22.6 \%$ & \\
\hline (Lateral) & $0.0 \%$ & $3.8 \%$ & & $0.0 \%$ & $4.5 \%$ & & $0.0 \%$ & $4.1 \%$ & & $2.1 \%$ & $1.9 \%$ & \\
\hline ( $\geq$ two territories) & $18.8 \%$ & $19.2 \%$ & & $18.2 \%$ & $18.2 \%$ & & $18.2 \%$ & $18.4 \%$ & & $27.7 \%$ & $7.5 \%$ & \\
\hline S1Q3T3 & $12.5 \%$ & $19.2 \%$ & 0.4 & $18.2 \%$ & $22.7 \%$ & 0.3 & $18.2 \%$ & $22.4 \%$ & 0.4 & $21.3 \%$ & $17.0 \%$ & 0.4 \\
\hline Premature ventricular complexes & $12.5 \%$ & $26.9 \%$ & 0.2 & $25.0 \%$ & $18.2 \%$ & 0.3 & $25.0 \%$ & $18.4 \%$ & 0.3 & $25.5 \%$ & $20.6 \%$ & 0.4 \\
\hline Premature atrial complexes & $6.3 \%$ & $15.4 \%$ & 0.3 & $15.9 \%$ & $9.1 \%$ & 0.3 & $15.9 \%$ & $10.2 \%$ & 0.3 & $12.8 \%$ & $13.2 \%$ & 0.9 \\
\hline Poor $\mathrm{R}$ wave progression & $18.8 \%$ & $11.5 \%$ & 0.3 & $18.2 \%$ & $11.4 \%$ & 0.2 & $18.2 \%$ & $10.2 \%$ & 0.2 & $17.0 \%$ & $11.3 \%$ & 0.2 \\
\hline \multicolumn{13}{|l|}{ Rhythm on admission } \\
\hline Sinus rhythm & $56.3 \%$ & $59.6 \%$ & \multirow[t]{3}{*}{0.1} & $61.4 \%$ & $45.5 \%$ & \multirow[t]{3}{*}{0.2} & $61.4 \%$ & $46.9 \%$ & \multirow[t]{3}{*}{0.34} & $59.6 \%$ & $52.8 \%$ & \multirow[t]{3}{*}{0.7} \\
\hline Sinus tachycardia & $25.0 \%$ & $36.5 \%$ & & $27.3 \%$ & $43.2 \%$ & & $27.3 \%$ & $40.8 \%$ & & $29.8 \%$ & $35.8 \%$ & \\
\hline Atrial fibrillation & $18.8 \%$ & $3.8 \%$ & & $11.4 \%$ & $11.4 \%$ & & $11.4 \%$ & $12.2 \%$ & & $10.6 \%$ & $11.3 \%$ & \\
\hline \multicolumn{13}{|l|}{ Rhythm during admission } \\
\hline Atrial arrhythmia & $18.8 \%$ & $17.3 \%$ & 0.57 & $20.5 \%$ & $20.5 \%$ & 0.6 & $20.5 \%$ & $22.4 \%$ & 0.5 & $14.9 \%$ & $28.3 \%$ & 0.08 \\
\hline Atrial fibrillation & $18.8 \%$ & $13.5 \%$ & 0.4 & $20.5 \%$ & $18.2 \%$ & 0.5 & $20.5 \%$ & $16.3 \%$ & 0.4 & $12.8 \%$ & $22.6 \%$ & 0.1 \\
\hline Atrial flutter & $6.3 \%$ & $1.9 \%$ & 0.4 & $2.3 \%$ & $6.8 \%$ & 0.3 & $2.3 \%$ & $8.2 \%$ & 0.2 & $4.3 \%$ & $7.5 \%$ & 0.3 \\
\hline Atrial tachycardia & $6.3 \%$ & $3.8 \%$ & 0.5 & $0.0 \%$ & $4.5 \%$ & 0.2 & $0.0 \%$ & $6.1 \%$ & 0.1 & $0.0 \%$ & $5.7 \%$ & 0.1 \\
\hline Sinus bradycardia & $18.6 \%$ & $9.6 \%$ & 0.2 & $18.2 \%$ & $9.1 \%$ & 0.1 & $18.2 \%$ & $8.2 \%$ & 0.1 & $12.8 \%$ & $13.2 \%$ & 0.5 \\
\hline Sinus tachycardia & $81.3 \%$ & $92.3 \%$ & 0.2 & $72.7 \%$ & $90.9 \%$ & 0.02 & $72.7 \%$ & $89.8 \%$ & 0.03 & $74.5 \%$ & $86.8 \%$ & 0.1 \\
\hline Left atrial abnormality & $6.3 \%$ & $2.0 \%$ & 0.4 & $2.4 \%$ & $9.1 \%$ & 0.1 & $2.4 \%$ & $8.2 \%$ & 0.2 & $2.2 \%$ & $7.5 \%$ & 0.2 \\
\hline Right atrial abnormality & $0.0 \%$ & $7.8 \%$ & 0.3 & $11.9 \%$ & $4.5 \%$ & 0.1 & $11.9 \%$ & $4.1 \%$ & 0.1 & $6.7 \%$ & $7.5 \%$ & 0.5 \\
\hline Left axis deviation & $31.3 \%$ & $23.1 \%$ & \multirow[t]{2}{*}{0.7} & $23.3 \%$ & $31.8 \%$ & \multirow[t]{2}{*}{0.4} & $23.3 \%$ & $28.6 \%$ & \multirow[t]{2}{*}{0.8} & $25.5 \%$ & $23.1 \%$ & \multirow[t]{2}{*}{0.9} \\
\hline Right axis deviation & $0.0 \%$ & $1.9 \%$ & & $2.3 \%$ & $0.0 \%$ & & $2.3 \%$ & $2.0 \%$ & & $2.1 \%$ & $1.9 \%$ & \\
\hline Right bundle branch block (RBBB) & $6.3 \%$ & $1.9 \%$ & 0.4 & $6.8 \%$ & $2.3 \%$ & 0.3 & $6.8 \%$ & $2.0 \%$ & 0.3 & $4.3 \%$ & $3.8 \%$ & 0.6 \\
\hline Incomplete RBBB & $6.3 \%$ & $1.9 \%$ & 0.4 & $2.3 \%$ & $4.5 \%$ & 0.5 & $2.3 \%$ & $4.1 \%$ & 0.5 & $2.1 \%$ & $3.8 \%$ & 0.5 \\
\hline Q wave & $18.8 \%$ & $23.1 \%$ & 0.5 & $27.3 \%$ & $29.5 \%$ & 0.5 & $27.3 \%$ & $28.6 \%$ & 0.5 & $25.5 \%$ & $28.3 \%$ & 0.4 \\
\hline Inferior Q wave & $12.5 \%$ & $23.1 \%$ & \multirow[t]{3}{*}{0.1} & $18.2 \%$ & $29.5 \%$ & \multirow[t]{3}{*}{0.1} & $18.2 \%$ & $28.6 \%$ & \multirow[t]{3}{*}{0.1} & $19.1 \%$ & $24.5 \%$ & \multirow[t]{3}{*}{0.1} \\
\hline Anterior Q wave & $0.0 \%$ & $0.0 \%$ & & $2.3 \%$ & $0.0 \%$ & & $2.3 \%$ & $0.0 \%$ & & $0.0 \%$ & $3.8 \%$ & \\
\hline Q wave in $\geq 2$ territories & $6.3 \%$ & $0.0 \%$ & & $6.8 \%$ & $0.0 \%$ & & $6.8 \%$ & 0.05 & & $6.4 \%$ & $0.0 \%$ & \\
\hline Low voltage & $12.5 \%$ & $15.4 \%$ & 0.56 & $18.2 \%$ & $11.4 \%$ & 0.3 & $18.2 \%$ & $12.2 \%$ & 0.3 & $23.4 \%$ & $9.4 \%$ & 0.051 \\
\hline Left ventricular hypertrophy & $6.3 \%$ & $11.5 \%$ & 0.4 & $6.8 \%$ & $25.0 \%$ & 0.01 & $6.8 \%$ & $22.4 \%$ & 0.03 & $19.1 \%$ & $11.3 \%$ & 0.2 \\
\hline Left anterior fascicular block & $25.0 \%$ & $3.8 \%$ & 0.02 & $13.6 \%$ & $13.6 \%$ & 0.6 & $13.6 \%$ & $12.2 \%$ & 0.5 & $17.0 \%$ & $7.5 \%$ & 0.1 \\
\hline Left posterior fascicular block & $0.0 \%$ & $1.9 \%$ & 0.7 & $0.0 \%$ & $0.0 \%$ & - & $0.0 \%$ & $2.0 \%$ & 0.5 & $0.0 \%$ & $1.9 \%$ & 0.5 \\
\hline Bi-fascicular block & $6.3 \%$ & $1.9 \%$ & 0.4 & $6.8 \%$ & $2.3 \%$ & 0.3 & $6.8 \%$ & $2.0 \%$ & 0.2 & $4.3 \%$ & $3.8 \%$ & 0.6 \\
\hline Normal QRS width & $93.8 \%$ & $98.1 \%$ & 0.4 & $90.9 \%$ & $97.7 \%$ & 0.1 & $90.9 \%$ & $98.0 \%$ & 0.1 & $95.7 \%$ & $94.3 \%$ & 0.5 \\
\hline
\end{tabular}

Table 4S : Baseline characteristics of our cohort of 100 patients diagnosed with COVID-19 
medRxiv preprint doi: https://doi.org/10.1101/2021.01.29.21250407; this version posted February 1, 2021. The copyright holder for this preprint (which was not certified by peer review) is the author/funder, who has granted medRxiv a license to display the preprint in perpetuity.

\begin{tabular}{|c|c|c|c|c|}
\hline Variable & $\mathrm{N}=\mathbf{1 0 0}$ & $\begin{array}{c}\text { Survivors } \\
\mathrm{N}=52\end{array}$ & $\begin{array}{c}\text { Non-survivors } \\
\mathrm{N}=48\end{array}$ & P-value \\
\hline Age (median, IQR) & $66(57-74)$ & $65(47.5-71)$ & $68.5(60-75)$ & 0.1 \\
\hline \multicolumn{5}{|l|}{ Race } \\
\hline Male gender & $61 \%$ & $67.3 \%$ & $54.2 \%$ & 0.2 \\
\hline African Americans & $77 \%$ & $82.7 \%$ & $70.8 \%$ & \multirow[t]{3}{*}{0.3} \\
\hline White & $6 \%$ & $3.8 \%$ & $8.3 \%$ & \\
\hline Other & $17 \%$ & $13.5 \%$ & $20.8 \%$ & \\
\hline Body mass index $\mathrm{kg} / \mathrm{m}^{2}$ & $31(26.1-39)$ & $30.9(25.9-36.8)$ & $31.2(26.6-41)$ & 0.2 \\
\hline Hypertension & $71 \%$ & $71.2 \%$ & $70.8 \%$ & 1.0 \\
\hline Diabetes Mellitus & $40 \%$ & $30.8 \%$ & $50 \%$ & 0.05 \\
\hline Dyslipidemia & $41 \%$ & $40.4 \%$ & $41.7 \%$ & 0.9 \\
\hline Chronic/end-stage renal disease & $19 \%$ & $21.2 \%$ & $16.7 \%$ & 0.6 \\
\hline Lung disease & $26 \%$ & $23.1 \%$ & $29.2 \%$ & 0.5 \\
\hline Obstructive sleep apnea & $11 \%$ & $5.8 \%$ & $16.7 \%$ & 0.08 \\
\hline Cancer & $15 \%$ & $13.5 \%$ & $16.7 \%$ & 0.6 \\
\hline Immunological & $7 \%$ & $7.6 \%$ & $6.2 \%$ & 0.6 \\
\hline Mental disorder & $12 \%$ & $15.3 \%$ & $8.3 \%$ & 0.3 \\
\hline Neurological disorder & $11 \%$ & $11.5 \%$ & $10.4 \%$ & 0.9 \\
\hline Coronary artery disease & $12 \%$ & $17.3 \%$ & $6.3 \%$ & 0.08 \\
\hline Systolic heart failure & $10 \%$ & $15.4 \%$ & $4.2 \%$ & 0.06 \\
\hline Atrial fibrillation/Atrial flutter & $10 \%$ & $15.4 \%$ & $4.2 \%$ & 0.06 \\
\hline Troponin (Peak) & $38.5(10-187.2)$ & $23.5(9-185)$ & $43.5(18.75-187.2)$ & 0.2 \\
\hline Brain Natriuretic peptide (BNP) & $130(52.5-411.5)$ & $123(35.25-261)$ & $158(56-1034)$ & 0.5 \\
\hline Ferritin & $971(418-1952.5)$ & $884(383-1345)$ & $1035(522-2802)$ & 0.04 \\
\hline D-dimer & $2.2(1.12-21.35)$ & $1.34(0.5-4.3)$ & $4.9(1.6-35.2)$ & 0.002 \\
\hline C-reactive protein (CRP) & $175.0(81.7-292.0)$ & $171.5(66.5-267.5)$ & $207.5(95.7-323.0)$ & 0.2 \\
\hline \multicolumn{5}{|l|}{ Treatment } \\
\hline $\begin{array}{l}\text { No Hydroxychloroquine (HY)/ } \\
\text { Azithromycin(AZ)/Doxycycline }\end{array}$ & $12(12.0 \%)$ & $4(7.7 \%)$ & $8(16.7 \%)$ & \multirow[t]{4}{*}{0.02} \\
\hline HY only & $19(19.0 \%)$ & $9(17.3 \%)$ & $10(20.8 \%)$ & \\
\hline HY \& AZ & $40(40.0 \%)$ & $17(32.7 \%)$ & $23(47.9 \%)$ & \\
\hline HY \& Doxycycline & $29(29.0 \%)$ & $22(42.3 \%)$ & $7(14.6 \%)$ & \\
\hline Tocilizumab & $5 \%$ & $0 \%$ & $10.4 \%$ & 0.02 \\
\hline Steroids & $53 \%$ & $50 \%$ & $56.3 \%$ & 0.5 \\
\hline L-ascorbic acid & $24 \%$ & $23.1 \%$ & $25 \%$ & 0.8 \\
\hline Zinc & $16 \%$ & $15.4 \%$ & $16.7 \%$ & 0.9 \\
\hline Anticoagulation & $32 \%$ & $25 \%$ & $39.6 \%$ & 0.1 \\
\hline Single antiplatelet drug & $33 \%$ & $44.2 \%$ & $20.8 \%$ & \multirow[t]{2}{*}{0.03} \\
\hline Dual antiplatelet therapy & $7 \%$ & $3.8 \%$ & $10.4 \%$ & \\
\hline Statins & $38 \%$ & $38.5 \%$ & $37.5 \%$ & 0.9 \\
\hline$\geq$ Vasopressor & $37 \%$ & $5.8 \%$ & $70.8 \%$ & $<0.001$ \\
\hline Beta blockers & $41 \%$ & $50 \%$ & $31.3 \%$ & 0.06 \\
\hline Calcium channel blocker & $8 \%$ & $7.7 \%$ & $8.3 \%$ & 0.6 \\
\hline Amiodarone & $7 \%$ & $3.8 \%$ & $10.4 \%$ & 0.2 \\
\hline Corrected QT(QTc) on admission (milliseconds) & $443(410.2-472.7)$ & 435 ( 404.5-476.7) & $447(416.2-465.7)$ & 0.8 \\
\hline Smoking & $30 \%$ & $36.5 \%$ & $22.9 \%$ & 0.1 \\
\hline Alcohol & $20 \%$ & $23.1 \%$ & $16.7 \%$ & 0.4 \\
\hline Illicit drugs & $9 \%$ & $15.4 \%$ & $2.1 \%$ & 0.02 \\
\hline Potassium trough & $3.6(3.3-4.0)$ & $3.7(3.3-3.9)$ & $3.6(3.4-4)$ & 0.4 \\
\hline Potassium peak & $4.75(4.32-5.5)$ & $4.75(4.3-5.5)$ & $4.75(4.4-5.4)$ & 0.7 \\
\hline Magnesium trough & $1.9(1.7-2.1)$ & $1.9(1.72-2.1)$ & $2(1.7-2.2)$ & 0.5 \\
\hline Magnesium peak & $2.4(2.2-2.7)$ & $2.3(2.1-2.6)$ & $2.6(2.3-2.87)$ & 0.001 \\
\hline Sodium trough & $134(131-137)$ & $134(132-136)$ & $135(130-138)$ & 0.5 \\
\hline Sodium peak & $143(140-147)$ & $142(140-145)$ & $145(140.2-148.7)$ & 0.03 \\
\hline Calcium trough & $7.9(7.4-8.3)$ & $8.1(7.6-8.4)$ & $7.65(7.1-8.2)$ & 0.005 \\
\hline Calcium peak & $9.0(8.5-9.4)$ & $9.2(8.9-9.6)$ & $8.7(8.5-9.2)$ & 0.001 \\
\hline Phosphorus trough & $2.7(2.2-3.8)$ & $2.6(2.1-3.2)$ & $3(2.2-4.3)$ & 0.2 \\
\hline Phosphorus peak & $4.5(3.4-6.7)$ & $3.7(3-4.7)$ & 5.3(4.1-7.7) & $<0.001$ \\
\hline Intensive care unit (ICU) admission & $48(48.0 \%)$ & $8(21.6 \%)$ & $40(63.5 \%)$ & $<0.001$ \\
\hline ICU length of stay & $3(0-8.9)$ & $0(0-3.75)$ & $7(2.2-13.1)$ & $<0.001$ \\
\hline Hospital length of stay & $10.4(6.7-16)$ & $10.6(7.07-16.4)$ & $9.4(5.7-15.6)$ & 0.5 \\
\hline
\end{tabular}

